\title{
Simulation of people's movements on floors using social force model
}

\author{
Ahmed S. Mohammed and Aleksandar Pavic \\ Vibration Engineering Section, College of Engineering, Mathematics and Physical Sciences, University \\ of Exeter, Kay Building, North Park Road, Exeter, EX4 4QF, U.K.
}

\begin{abstract}
Vibration serviceability assessment of floors has been traditionally based on a scenario of a single person walking along a path which will generate maximum vibration level. This is due to the difficulty of predicting the real positions and paths of the walking people. With such a design scenario, it is possible to obtain calculated responses, which could be both over- or underestimated, depending on the specifics. This could be due to considering only one person walking along one walking path in the simulations. This aspect in the design guidelines could be improved if realistic modelling of people's movements is utilised. Hence, this paper examines the performance of the social force model to simulate the behaviour of people's movements on floors. This method has been widely used to model a crowd of people in evacuation and panic situations. However, it has been reported in the literature that this approach could be used to model people's movements in normal situations as well. The simulation carried out in this paper focuses on the interaction between walking people themselves and between walking people and the surrounding boundaries in typical office floors. The results show that reasonable and realistic behaviour of the floor occupants could be obtained using the social force model. Furthermore, utilising the 'heatmap' can help the designers to visualise and obtain information about the proportion of time spent by walking individuals at various points on the floor. This approach can be adopted in a more realistic procedure for the vibration serviceability assessment of floors.
\end{abstract}

Keywords: Social force model, people's movements, walking path, floors, vibration serviceability.

\section{Introduction}

Vibration serviceability assessment due to human walking excitation is increasingly governing the design of building floors [1]. The corresponding criterion is traditionally based on the assumption of a single person walking in a straight line related to the maximum vibration level [2-5]. In reality, people's movement on building floors is a complex process that involves multiple people where the walking behaviour of each person is affected by other people and the surrounding boundaries. Therefore, modelling natural people's movements on floors can add a significant improvement towards more realistic vibration serviceability assessment. This is required by the very nature of the serviceability limit state analysis which should take into account as realistically as possible the natural day-to-day operational condition of the structure.

Modelling people's movement in indoor environments is widely utilised in building evacuation and crowd safety analysis, planning of smart and energy efficient buildings and video games [6-8]. These models can be divided into two categories: macroscopic models, which are based on the similarity between crowd movements and the flow of fluids, and microscopic models, which focus on the behaviour of each person [7,9]. The latter case is preferable for normal scenarios where low density of people is expected [10]. One of the most widely used models for this purpose is the social force model [10]. While the theory behind it is mostly based on 'panic scenarios', it can also be used for normal situations [10].

This paper summarises and examines how the social force model can simulate the behaviour of walking people on floors in normal situations. The model is used within an agent-based modelling (ABM) framework designed for this purpose. The performance is demonstrated using computer simulation of people walking on a typical office floor. 


\section{Methodology}

One of the most efficient frameworks to simulate people's movements is the ABM approach. Using this method, each person thinks and behaves individually, but interacts with surrounding people and the environment. This interaction can be simulated using the social force model. The collective behaviour of walking people emerges from these individual interactions [10]. The key elements of modelling people's movements can be summarised as follows [6]:

- Decision-making. This governs the general behaviour of each person and it involves the "thinking" process and the acts the person decides to perform (e.g. making a decision to move from the current position to another position).

- Pathfinding. After a decision has been made, to move to another position, the person needs to find the shortest available path which s/he can follow to reach the destination.

- Steering. This guides the walking person to follow the pre-decided path.

- Perception. During walking, each person need to sense the surrounding people, obstacles and walls. This will guide the walking person during walking to prevent collision with the surrounding people or boundaries.

The design of the decision-making part can vary depending on the objectives of the simulations and the degree of detail needed. To design the pathfinding part, different strategies can be employed. A simple and efficient strategy is to subdivide the area and map it into nodes. These nodes are used to guide the pedestrians until they reach their destination [7]. Based on this, an algorithm for the shortest path can be implemented. During walking, the laws of the social force model control the steering and perception of individuals as explained in the next section.

\section{Social force model}

The social force model assumes that the internal and external stimuli acting on walking people can be represented by physical force vectors, which are known as social forces [11]. The resultant social force, which is the summation of all social forces, can be used to determine the walking acceleration, walking speed and the position of pedestrians. This is based on Newton's Second Law which states that the force $(f)$ acting on an object is equal to the mass $(m)$ of the object multiplied by its acceleration $(a)$ (i.e. $f=m \cdot a)$. Similarly, the vector of the walking acceleration $\left(\vec{a}_{p_{1}}\left(t_{i}\right)\right)$ of a walking pedestrian $p_{1}$ at time step $t_{i}$ can be computed if the corresponding body mass $\left(m_{p_{1}}\right)$ and the vector of the resultant social force $\left(\vec{f}_{p_{1}}\left(t_{i}\right)\right)$ are known, and as expressed in Eq. (1) [10,12,13].

$$
\vec{a}_{p_{1}}\left(t_{i}\right)=\frac{\vec{f}_{p_{1}}\left(t_{i}\right)}{m_{p_{1}}}
$$

The walking acceleration can be integrated to obtain the walking speed $\left(\vec{v}_{p_{1}}\left(t_{i+1}\right)\right)$ and the position $\left(\vec{x}_{p_{1}}\left(t_{i+1}\right)\right)$ of the pedestrian at time step $t_{i+1}$ as described in Eq. (2) and Eq. (3), respectively.

$$
\begin{aligned}
& \vec{v}_{p_{1}}\left(t_{i+1}\right)=\vec{v}_{p_{1}}\left(t_{i}\right)+\vec{a}_{p_{1}}\left(t_{i}\right) \Delta t, \\
& \vec{x}_{p_{1}}\left(t_{i+1}\right)=\vec{x}_{p_{1}}\left(t_{i}\right)+\vec{v}_{p_{1}}\left(t_{i}\right) \Delta t+\frac{1}{2} \vec{a}_{p_{1}}\left(t_{i}\right) \Delta t^{2},
\end{aligned}
$$

where, $\Delta t$ is the duration of the time step [s]. In Eq. (2) and Eq. (3), $\vec{v}_{p_{1}}\left(t_{i}\right)$ and $\vec{x}_{p_{1}}\left(t_{i}\right)$ are known from the previous steps or initial conditions when $t_{i}=0$.

The resultant social force $\left(\vec{f}_{p_{1}}\left(t_{i}\right)\right)$ can be obtained by computing the summation of three force vectors: driving force $\left(\vec{f}_{p_{1}, d r i}\left(t_{i}\right)\right)$, inter-pedestrian force $\left(\vec{f}_{p_{1}, \text { ped }}\left(t_{i}\right)\right)$ and boundary force $\left(\vec{f}_{p_{1}, \text { bound }}\left(t_{i}\right)\right)$ as described in Eq. (4) and shown in Fig. 1.

\subsection{Driving force}

$$
\vec{f}_{p_{1}}\left(t_{i}\right)=\vec{f}_{p_{1}, \text { dri }}\left(t_{i}\right)+\vec{f}_{p_{1}, \text { ped }}\left(t_{i}\right)+\vec{f}_{p_{1}, \text { bound }}\left(t_{i}\right),
$$

The driving force accounts for the desire of pedestrians to walk towards a target (Fig. 1). During walking, pedestrians try to maintain a desired walking speed. The actual speed may deviate from that due to the obstruction caused by other people and boundaries. Hence, the driving force stimulates the pedestrians to walk at the desired walking speed as described in Eq. (5) [10,11].

$$
\vec{f}_{p_{1}, d r i}\left(t_{i}\right)=m_{p_{1}} \frac{v_{d} \vec{e}_{p_{1}}-\vec{v}_{p_{1}}\left(t_{i}\right)}{t_{r}}
$$




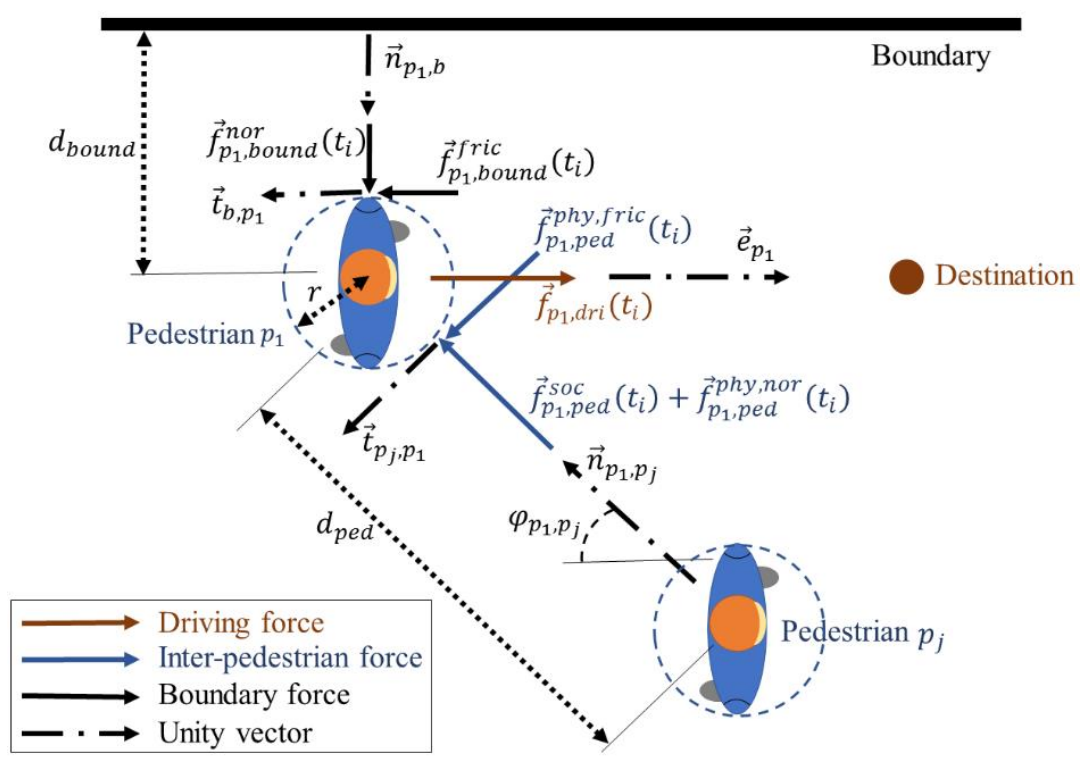

Fig. 1. Decomposition of the social forces acting on pedestrian $p_{1}[13,14]$.

where, $v_{d}$ is a scalar value describing the desired walking speed $[\mathrm{m} / \mathrm{s}], \vec{e}_{p_{1}}$ is a unit vector which represents the direction of movement (Fig. 1) and $t_{r}$ is the relaxation time (Table 1). The relaxation time refers to the time needed by pedestrians to adapt from the actual $\left(\vec{v}_{p_{1}}\left(t_{i}\right)\right)$ to the desired walking speed $\left(v_{d} \vec{e}_{p_{1}}\right)[10]$.

Table 1. Parameters of the social force model [11].

\begin{tabular}{cl}
\hline Parameter & Value \\
\hline$t_{r}$ & $0.5 \mathrm{~s}$ \\
$r$ & $0.3 \mathrm{~m}$ \\
$A$ & $2000 \mathrm{~N}$ \\
$B$ & $0.08 \mathrm{~N}$ \\
$\lambda$ & 0.2 \\
$C$ & $120,000 \mathrm{~kg} \mathrm{~s}^{-2}$ \\
$D$ & $240,000 \mathrm{~kg} \mathrm{~s}^{-2}$ \\
\hline
\end{tabular}

\subsection{Inter-pedestrian force}

The interaction among pedestrians has two components: social and physical interactions (Fig. 1). Social interaction describes the tendency of pedestrians to keep a certain distance from other pedestrians and it can be represented by a repulsive social force $\left(\vec{f}_{p_{1}, \text { ped }}^{\text {soc }}\left(t_{i}\right)\right)$. Physical interaction accounts for physical contact when pedestrians are very close to each other and it can be represented by a force $\left(\vec{f}_{p_{1}, \text { ped }}^{\text {phy }}\left(t_{i}\right)\right)$ with two terms: normal $\left(\vec{f}_{p_{1}, \text { ped }}^{\text {phy,nor }}\left(t_{i}\right)\right)$ and sliding friction $\left(\vec{f}_{p_{1}, \text { ped }}^{\text {phy,fric }}\left(t_{i}\right)\right)$ forces. These forces are shown in Fig. 1 and they can be obtained using the following equations [10].

$$
\begin{aligned}
& \vec{f}_{p_{1}, \text { ped }}\left(t_{i}\right)=\vec{f}_{p_{1}, \text { ped }}^{\text {soc }}\left(t_{i}\right)+\vec{f}_{p_{1}, \text { ped }}^{\text {phy }}\left(t_{i}\right), \\
& \vec{f}_{p_{1}, \text { ped }}^{\text {soc }}\left(t_{i}\right)=\sum_{j=1}^{n} A e^{\frac{2 r-d_{\text {ped }}}{B}} \vec{n}_{p_{1}, p_{j}}\left(\lambda+(1-\lambda) \frac{1+\cos \left(\varphi_{p_{1}, p_{j}}\right)}{2}\right), \\
& \vec{f}_{p_{1}, \text { ped }}^{\text {phy }}\left(t_{i}\right)=\vec{f}_{p_{1}, \text { ped }}^{\text {hhy,nor }}\left(t_{i}\right)+\vec{f}_{p_{1}, \text { ped }}^{\text {hhy, fric }}\left(t_{i}\right), \\
& \vec{f}_{p_{1}, \text { ped }}^{\text {phy,nor }}\left(t_{i}\right)=\sum_{j=1}^{n} C \theta_{\text {ped }} \vec{n}_{p_{1}, p_{j}}, \\
& \vec{f}_{p_{1}, \text { ped }}^{\text {phy,fric }}\left(t_{i}\right)=\sum_{j=1}^{n} D \theta_{\text {ped }}\left\langle\overrightarrow{\Delta v}_{p_{1}, p_{j}} \cdot \vec{t}_{p_{j}, p_{1}}\right\rangle \vec{t}_{p_{j}, p_{1}}, \\
& \theta_{\text {ped }}=\left\{\begin{array}{c}
2 r-d_{\text {ped }} \text { if } 2 r-d_{\text {ped }}>0 \\
0 \quad \text { if } 2 r-d_{\text {ped }} \leq 0
\end{array}\right.
\end{aligned}
$$




$$
\overrightarrow{\Delta v}_{p_{1}, p_{j}}=\vec{v}_{p_{1}}\left(t_{i}\right)-\vec{v}_{p_{j}}\left(t_{i}\right)
$$

where, $A$ is the interaction strength (Table 1), $r$ is the radius of the pedestrian (Table 1), $d_{\text {ped }}$ is the distance between the centre of masses of pedestrians $p_{1}$ and $p_{j}[\mathrm{~m}]$ (where, $j$ denotes the number of another pedestrian), $n$ is the number of pedestrians excluding the pedestrian $p_{1}, B$ is the range of repulsive interaction (Table 1), $\vec{n}_{p_{1}, p_{j}}$ is a unit vector pointing from an pedestrian $p_{j}$ to pedestrian $p_{1}$ (Fig. 1), $\varphi_{p_{1}, p_{j}}$ is the angle between the direction of motion and the direction of the force exerted by pedestrian $p_{j}$ on pedestrian $p_{1}$ (Fig. 1), $\lambda$ is the potential factor which is related to the fact that the pedestrians in front of pedestrian $p_{1}$ have more effect than those behind this pedestrian (Table 1), $C$ is the body force strength due to contact (Table 1), $\theta_{\text {ped }}$ is a parameter which depends on the existence of physical contact between pedestrians $p_{1}$ and $p_{j}$ as calculated in Eq. (11) [m], $D$ is the sliding friction strength (Table 1), $\overrightarrow{\Delta v}_{p_{1}, p_{j}}$ is the relative walking speed between pedestrians $p_{1}$ and $p_{j}$ and it can be calculated using Eq. (12) [m/s], $\vec{t}_{p_{j}, p_{1}}$ is a unit vector perpendicular to $\vec{n}_{p_{1}, p_{j}}$ (Fig. 1) and \langle\rangle denotes a scalar product of two vectors.

\subsection{Boundary force}

The interaction between pedestrians and boundaries can be treated in a similar way to the interaction among pedestrians (Fig. 1). Hence, the force corresponding to this interaction has two components, normal $\left(\vec{f}_{p_{1}, \text { bound }}^{\text {nor }}\left(t_{i}\right)\right)$ and sliding friction $\left(\vec{f}_{p_{1}, \text { bound }}^{\text {fric }}\left(t_{i}\right)\right)$ forces as shown in Fig. 1 and described in the following equations [10].

$$
\begin{aligned}
& \vec{f}_{p_{1}, \text { bound }}\left(t_{i}\right)=\vec{f}_{p_{1}, \text { bound }}^{\text {nor }}\left(t_{i}\right)+\vec{f}_{p_{1}, \text { bound }}^{\text {fric }}\left(t_{i}\right), \\
& \vec{f}_{p_{1}, \text { bound }}^{\text {nor }}\left(t_{i}\right)=\sum_{j=1}^{n} A e^{\frac{2 r-d_{\text {bound }}}{B}} \vec{n}_{p_{1}, b}+C \theta_{\text {bound }} \vec{n}_{p_{1}, b}, \\
& \vec{f}_{p_{1}, \text { bound }}^{\text {fric }}\left(t_{i}\right)=\sum_{j=1}^{n} D \theta_{\text {bound }}\left\langle\vec{v}_{p_{1}}\left(t_{i}\right) \cdot \vec{t}_{b, p_{1}}\right\rangle \vec{t}_{b, p_{1}}, \\
& \theta_{\text {bound }}=\left\{\begin{array}{cl}
2 r-d_{\text {bound }} & \text { if } r-d_{\text {bound }}>0 \\
0 & \text { if } r-d_{\text {bound }} \leq 0
\end{array}\right.
\end{aligned}
$$

where, $d_{\text {bound }}$ is the distance between the centre of pedestrians and the boundary, $\vec{n}_{p_{1}, b}$ is a unit vector perpendicular to the boundary and pointing towards pedestrian $p_{1}$ (Fig. 1), $\theta_{\text {bound }}$ is a parameter which depends on the existence of a physical contact between pedestrian $p_{1}$ and the boundary as explained in Eq. (16) [m] and $\vec{t}_{b, p_{1}}$ is a unit vector perpendicular to $\vec{n}_{p_{1}, b}$ (Fig. 1).

\section{Demonstration}

\subsection{Description}

To demonstrate the performance of the social force model to simulate the behaviour of people's movements in normal situations, a typical office floor ( $21 \mathrm{~m}$ long and $14 \mathrm{~m}$ wide) is used (Fig. 2).

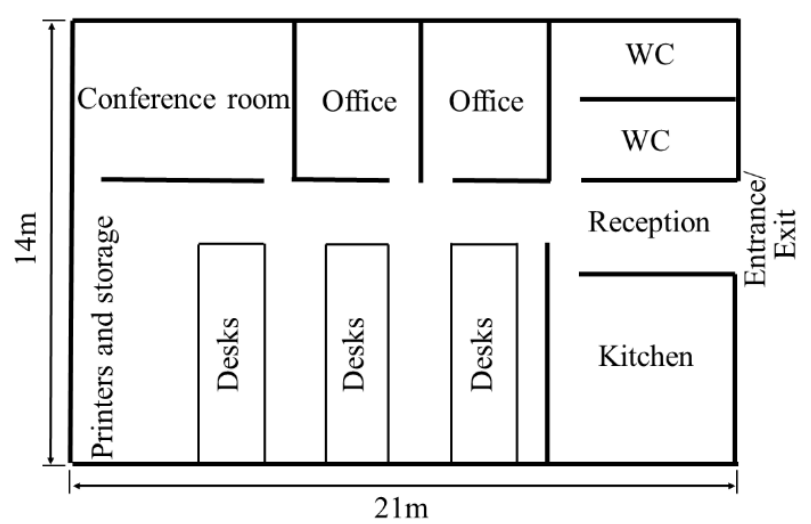

Fig. 2. Plan of a typical office floor used in the simulation. 
The floor comprises eight zones: two office rooms (two zones), a conference room, printing and storage space, three desks (one zone), a kitchen, a reception and a WC. The entrance/exit is situated near the reception (Fig. 2). One hour of the office floor usage by 20 people is simulated and, for simplicity, the following assumptions were made:

- The floor was divided into a grid of nodes with a spacing of $0.5 \mathrm{~m}$ between each of two horizontal or vertical nodes. This is to assist the pedestrians finding the shortest walking path and to guide them during walking.

- All floor occupants entered the floor within five minutes after the beginning of the simulation and they left the floor within the last five minutes. The exact entrance/exit times were assigned to each person randomly.

- During the simulation, the floor occupants moved independently to random nodes (positions) in any of the floor zones in Fig. 2. They spent five minutes at each node before moving to another random node in a random zone.

- The floor occupants interacted with each other and with the boundaries (walls and desks) during walking based on the social force model explained in the previous section.

- For simplicity, stationary people were assumed to have no effect in the social force model.

- A desired velocity of $1.5 \mathrm{~m} / \mathrm{s}$ and a body mass of $70 \mathrm{~kg}$ were assigned for all floor occupants.

- The time step of the simulation was $0.1 \mathrm{~s}$.

These assumptions were adopted to allow for a relatively high interaction among the floor occupants and between the floor occupants and the boundaries. A Matlab script was written to conduct this simulation and the results are shown below.

\subsection{Results}

There are different ways to present the distribution of walking people on the floor for the duration of the simulation. In this paper, this has been carried out as follows. The proportion of time spent by each walking person at each node in the floor is obtained. The summation of the proportions from all floor occupants is calculated and presented as a heatmap in Fig. 3. Therefore, this heatmap provides an indication of the positions where individuals are spending most of their time during walking. Similarly, other useful information can be obtained, for example: the probability of 1,2 or more people walking close together.

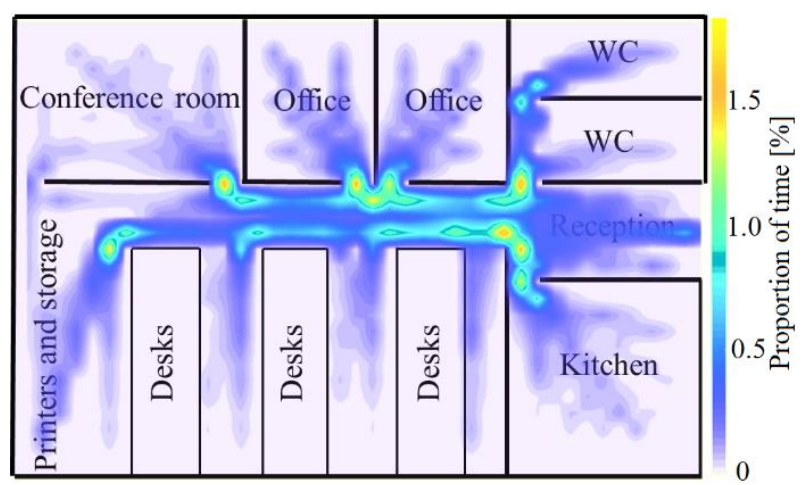

Fig. 3. The heatmap corresponding to the positions of the walking floor occupants.

In this figure, it is obvious how the boundary force has shaped the area where the floor occupants are walking and prevented them from walking into the walls or desks. A higher proportion of time spent by the walking people can be observed near the corners. This can be explained by the pedestrians reducing their walking speed when they approach corners (Fig. 3). A closer view on the behaviour of the floor occupants can be obtained by focusing on specific parts in the simulation. Fig. 4 and Fig. 5 show how the natural behaviour of walking people emerges when they interact with each other.

In Fig. 4, one person is entering while another person is leaving a room through the same door opening. Due to the space limit of the entrance, one person had to 'wait' until the other person went past before moving on. This waiting period is a result of that inter-pedestrian and boundary forces are opposing the driving force resulting in zero or low walking speed.

Another example of similar behaviour is given in Fig. 5, when one person had to make a temporary change in the walking path due to the entrance of another person into the corridor. This behaviour replicates the natural manoeuvres of walking people in indoor environments. The results indicate that the social force model can be used to simulate the natural behaviour of walking people in normal situations, as previously reported in the literature [10]. 
Time: 23 minute(s), 38.5 second(s)

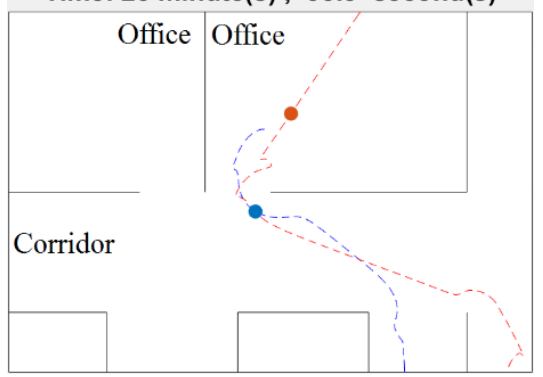

Time: 23 minute(s), 40 second(s)

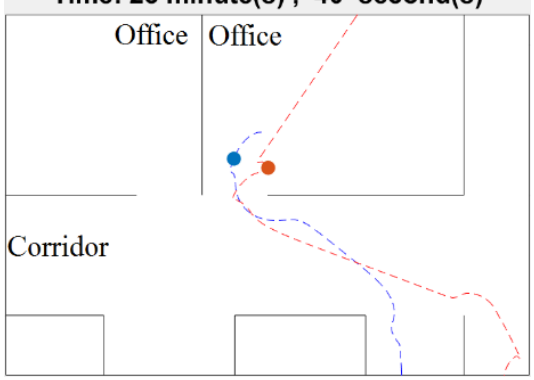

Time: 23 minute(s), 39 second(s)

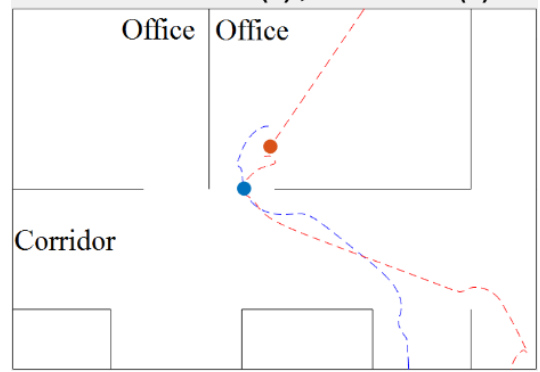

Time: 23 minute(s) , 40.5 second(s)

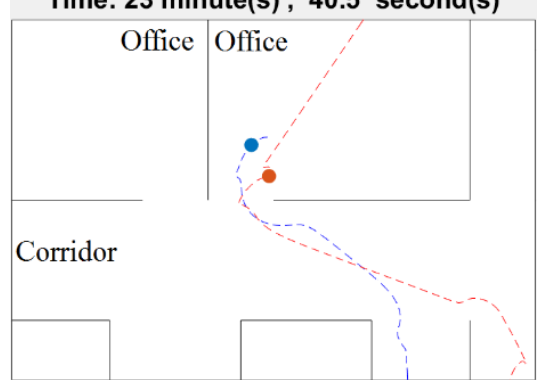

Time: 23 minute(s) , 39.5 second(s)

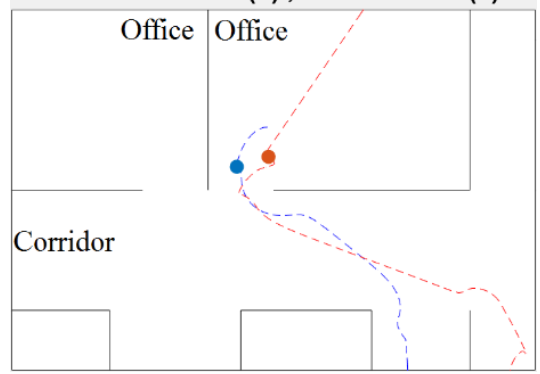

Time: 23 minute(s) , 41 second(s)

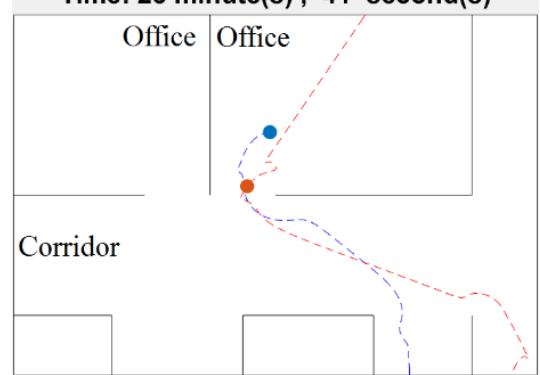

Fig. 4. Time-lapse snapshots of two floor occupants walking in opposite directions. One of them (blue circle) is entering while the other one (red circle) is leaving a room. The dashed lines represent their full walking paths.

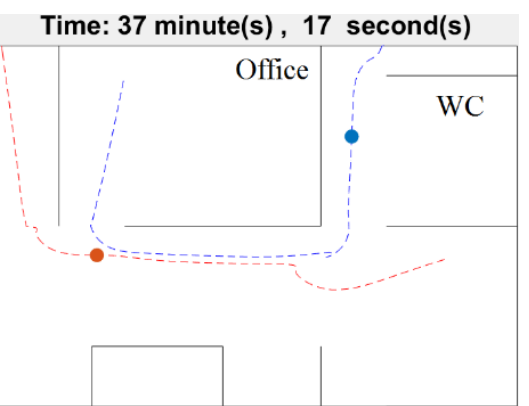

Time: 37 minute(s), 20 second(s)

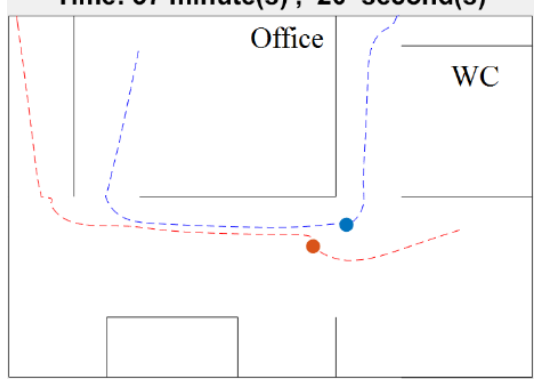

Time: 37 minute(s), 18 second(s)

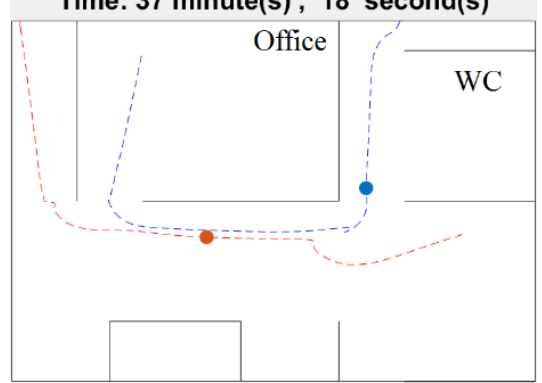

Time: 37 minute(s), 21 second(s)

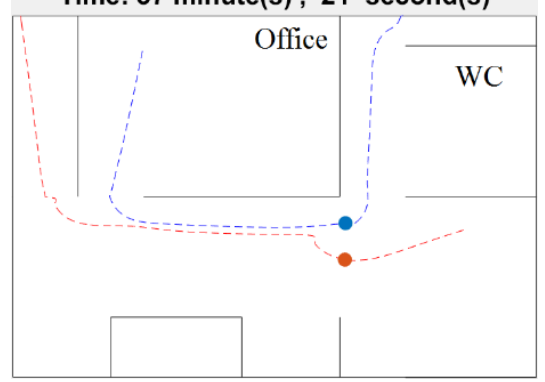

Time: 37 minute(s), 19 second(s)

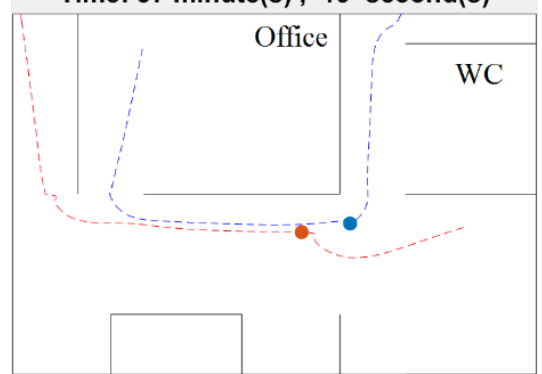

Time: 37 minute(s) , 22 second(s)

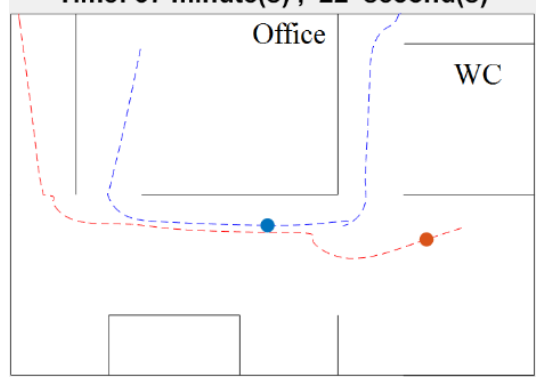

Fig. 5. Time-lapse snapshots of two floor occupants, one of them (red circle) is walking through the corridor while the other one (blue circle) is entering the corridor near the WC zone. The dashed lines represent their full walking paths.

\section{Conclusions}

The work presented in this paper indicates the potential to simulate the behaviour of multiple pedestrian movements in normal situations using the social force model. Realistic behaviour of the interacting people emerges from the 
realistic rules applied to each person. This is in-line with previous experimental studies regarding the performance of this model.

From the perspective of vibration serviceability, the detailed behaviour of an individual person traversing a floor may have no significant effect on the estimation of the floor vibration responses. However, the collective behaviour of walking people can be used for a much more realistic vibration serviceability assessment of the floors they walk on. For this purpose, the designers can use the information provided by the heatmap regarding the percentage of time various parts of the floor are occupied by a single person walking. This can be computed with other useful information, such as the frequency of occurrence of one or more people exciting the floor by walking close to a particular mode of vibration.

\section{Acknowledgements}

The authors are grateful for the College of Engineering, Mathematics and Physical Sciences in the University of Exeter for the financial support they provided for the first author and his $\mathrm{PhD}$ program. The authors would also like to acknowledge the financial support provided by the UK Engineering and Physical Sciences Research Council (EPSRC) for grant reference EP/K03877X/1 ('Modelling complex and partially identified engineering problemsApplication to the individualised multiscale simulation of the musculoskeletal system').

\section{References}

1. Brownjohn JMW, Racic V, Chen J. Universal response spectrum procedure for predicting walkinginduced floor vibration. Mech. Syst. Signal Process. , 1-15 (2015).

2. Pavic A, Willford MR. Vibration Serviceability of Post-tensioned Concrete Floors - CSTR43 App G. Append. G Post-Tensioned Concr. Floors Des. Handb. - Tech. Rep. 43. , 99-107 (2005).

3. Smith AL, Hicks SJ, Devine PJ. Design of floors for vibration - A new approach SCI P354, Revised Ed. The Steel Construction Institute.

4. Willford MR, Young P. A Design Guide for Footfall Induced Vibration of Structures - CCIP-016. The Concrete Centre, Slough.

5. Feldmann M, Heinemeyer C. Human Induced Vibration of Steel Structures - Vibration Design of Floors: Guideline. Research for coal and steel.

6. O'Connor S, Liarokapis F, Jayne C. Perceived realism of crowd behaviour with social forces. Proc. Int. Conf. Inf. Vis. 2015-Septe(August), 494-499 (2015).

7. Farina F, Fontanelli D, Garulli A, Giannitrapani A, Prattichizzo D. Walking ahead: The headed social force model. PLoS One. 12(1), 1-23 (2017).

8. Helbing D, Buzna B, Johansson A, Werner T. Self-organised pedestrian crowd dynamics: experiments, simulations, and design solution. Transp. Sci. 39(1), 1-24 (2005).

9. Moussaïd M, Perozo N, Garnier S, Helbing D, Theraulaz G. The walking behaviour of pedestrian social groups and its impact on crowd dynamics. PLoS One. 5(4), e10047 (2010).

10. Helbing D, Farkas I. Simulation of pedestrian crowds in normal and evacuation situations. Pedestr. evacuation Dyn. 21(January), 21-58 (2002).

11. Helbing D, Farkas I, Vicsek T. Simulating Dynamical Features of Escape Panic. 794(July), 487-490 (2000).

12. Carroll SP, Owen JS, Hussein MFM. Modelling crowd-bridge dynamic interaction with a discretely defined crowd. J. Sound Vib. 331(11), 2685-2709 (2012). 
13. Jimenez-Alonso JF, Saez A, Caetano E, Magalhães F. Vertical Crowd - Structure Interaction Model to Analyze the Change of the Modal Properties of a Footbridge. J. Bridg. Eng. (2016).

14. Helbing D, Molnár P. Social force model for pedestrian dynamics. Phys Rev E Stat Phys Plasmas Fluids Relat Interdiscip. 5(51), 4282-4286 (1995). 